\title{
Evaluating the effect of music intervention on hypertension
}

\author{
Vijay Mani Shankar ${ }^{1}$, B. Geethanjali ${ }^{1, *}$, Mahesh Veezhinathan ${ }^{1}$, \\ Jayaram Hariharakrishnan ${ }^{2}$, Nikhil Balakrishnan ${ }^{1}$ and L. Lakshmi ${ }^{3}$ \\ ${ }^{1}$ Department of Biomedical Engineering, and \\ ${ }^{2}$ Department of Information Technology, SSN College of Engineering, Old Mahabalipuram Road, Kalavakkam, Chennai 603 110, India \\ ${ }^{3}$ Sathyabama College of Nursing, Sathyabhama Institute of Science and Technology, Chennai 600 119, India
}

\begin{abstract}
The relevance of music in medical applications has been a subject of research for a long time. In the present study we evaluate the response of music interventions (Indian classical music) on elderly hypertensive participants. A randomized controlled assessment was done on 200 hypertensive participants, living in old age homes, and they were randomly segregated into two groups, viz. control and experimental. The means of the dependent variables (heart rate, respiratory rate (RR) and mean arterial pressure (MAP)) were compared using independent $t$-test between control and experimental groups. There was significant reduction in heart rate $(P<0.0001)$, MAP $(P<0.0001)$ and $\mathrm{RR}(P<0.0001)$ for the experimental group, whereas there was no change in heart rate $(P=0.113)$ and MAP $(P=0.364)$ for pre- and post-conditions (after one month) of the control group. However, significant reduction in mean $R R(P<0.005)$ was noted for control as well as for experimental group $(P<0.0001)$. The heart rate and MAP of the control group did not vary greatly under the influence of drugs. On the other hand, the difference in heart rate and MAP declined after music interventions for a period of one month. It is concluded that the experimental group preferred high valence and low arousal music which resulted in the reduction of heart rate and MAP.
\end{abstract}

Keywords: Hypertension, heart rate, mean blood pressure, music intervention, respiratory rate.

ACCORDING to the Indian Heart Association, hypertension is a long-term medical condition in which the blood pressure (BP) of arteries are constantly elevated. Public health estimates show that India accounts for approximately $60 \%$ of the world's heart disease burden, in spite of having less than $20 \%$ of the total population in the world. Heart disease is the most critical source of fatality and morbidity among South Asians ${ }^{1}$. The main treatment modality is pharmacological treatment, when lifestyle modification approaches fail to control BP. However, pharmacological treatment involves huge expenses and various hazardous effects, particularly in combination

*For correspondence. (e-mail: geethanjalib@ssn.edu.in) therapy $^{2}$. The current scenario demands more nonpharmacological treatments, such as music interventions, in the case of hypertension. Music therapy has been found to control BP during surgeries and in curing sleep disorders $^{3,4}$. Music plays a vital role in our lives ${ }^{5,6}$. Listening to music reduces the loneliness, boredom tension, influence the listener's moods, and fight loneliness ${ }^{7,8}$. Music therapy has become a significant element of complementary medicine ${ }^{9}$. Sarkamo et al. ${ }^{10}$ reported that music can be employed as a rehabilitative tool to cure various diseases. Various parts of the brain are engaged when we listen to music ${ }^{11}$, and its effects are reflected in the cardiovascular parameters, namely heart rate, respiratory rate (RR) and mean arterial pressure (MAP). Listening to music may induce a favourable effect on anxiety, systolic BP, heart rate, RR, sleep quality and pain in patients with coronary heart disease ${ }^{12}$. Music of interest is known to reduce depression and aid higher cognitive factors; it also improves molecular and structural flexibility in stroke patients recovering from brain injury ${ }^{13,14}$.

The American Music Therapy Association defines music therapy as the scientific and evidence-based implementation of music interventions to achieve desired targets within a therapeutic relationship by a trained expert $^{15}$.

Music interventions can also be cited as 'receptive music' 'music therapy' and 'music medicine'. Most of the studies on music intervention have used Western music as a stimulus. Listening to anonymous music from a different culture may curtail the emotional rewarding path than listening to indigenous known music ${ }^{16}$. In today's world hypertension has become a part of our lifestyle and treatment options have gained performance. In addition to the drugs prescribed, impact of music on the cardiovascular parameters is the main focus of the present study. We study the effects of preferred music intervention (South Indian classical music) on hypertensive patients using behavioural and cardiovascular parameters like RR, heart rate and MAP. The study hypothesizes that the selected music stimulus will have healthy outcome on physiological parameters changes. This can be used among elderly Indians as a possible therapy for improving the wellbeing of hypertensive patients. 


\section{Materials and methods}

\section{Selection of participants}

A controlled trial was done on 200 hypertensive participants, living in an old age home, and they were segregated into two groups, viz. experimental and control based on their interest in listening to Carnatic music during their leisure time ( $n=100$ in each group). The participants belonging to the control group did not listen to Carnatic music and were subjected only to drug therapy for hypertension. Participants in the experimental group listened to Carnatic music along with their regular drug therapy. The average age of the control group was $69.68 \pm 6.8$ years and that of the experimental group was $69.84 \pm 6.98$ years. The participants were all hypertensive patients with no history of seizures or any sensory impairment, and had no prior exposure to the concerned task. The experiment was conducted following the procurement of an informed consent from each of the participants.

\section{Stimulus}

Listening to Classical music augments the intensity of neurotransmitters. This leads to pleasant feeling and reduces depression ${ }^{17}$.

Malkauns is an old raga in Hindustani music and Hindolam as its Carnatic equivalent. Raga Malkauns belongs to the Bhairavi thaat or scale of Hindustani classical music. It is a pentatonic rage, i.e. its type is Audav-Audav and is sung in the late evening. The notes of this raga correspond to 'komal', where there is a smooth transition between the notes. The raga has a variation of presentations with brigatswaras and induces a sense of calmness; thus low arousal is observed in its elucidation. Malkauns is a serious, meditative raga, and is developed mostly in the lower octave (mandrasaptak) and in a slow tempo (vilambitlaya).

The uccha swaras or the consonants and the laya swaras or the dissonants are vibrantly spaced to create an enameling experience that is relaxing in nature.

Based on this, the experimental group was presented with a sitar-based musical piece in raga malkauns differing in the degree of dissonance.

Laya sampoorna (pleasant notes) is composed of excerpts of calming and peaceful rendition using sitar instrumental recorded in a professional studio under expert guidance from sound engineers. The stimulus had a run time of $15 \mathrm{~min}$ (range: $46.82-68.96 \mathrm{~s}$, M 5 $58.14 \mathrm{~s}$ ) and an average tempo of 2 beats/sec (bpm; M 5 $120.19 \mathrm{bpm}$, range: $64-154.12 \mathrm{bpm}$ ), and at times in shaukdhwani tempo consisting of 80 beats/sec with a lower pitch rate at 8 thaats/sec and higher pitch rate of 16 thaats/sec.
The single excerpts were controlled such that the effect did not increase or decrease with time in order to eliminate the difference in behavioural or electrophysiological measures between the first and second sets of laysa swaras of the excerpts due to tempo variations. At test for paired specimen, the first and second half of the stimuli showed invariant and sequentially understandable set of notes over the observed time frame.

\section{Experimental protocol}

The participants were segregated into two groups depending on their interest in Carnatic music. They followed regular diet with recommended drug dosage. The physiological parameters, namely RR, heart rate and MAP were initially measured for the 200 participants. The experimental group along with their regular activities listened to a particular Carnatic music, Raga Malkauns in the evening hours usually between $4: 30$ and $5: 30 \mathrm{pm}$, as it is an evening raga and most effective during the evening hours ${ }^{18}$. They were given earphones to listen to music and the volume level was adjusted according to individual preference. The music-listening session lasted for $15 \mathrm{~min}$ for the experimental group every day and this continued for 30 days. The participants from the experimental group were asked to rate their perceived emotions using selfassessment manikin scale on listening to Raga Malkauns ${ }^{19}$ and their mood before and after listening to music by the positive and negative affect (SAM) score (PANAS) scale ${ }^{20}$. The RR, heart rate and MAP of all the participants were recorded prior to music therapy (Figure 1). The experimental group was exposed to the stimulus for $15 \mathrm{~min}$ every day over a period of 30 days; their heart rate, RR and MAP were measured thereafter. On the contrary, the control group was not exposed to music therapy. Their RR, heart rate and MAP were also measured before the beginning of the protocol and after 30 days. The two sets of data were compared for analysis.

\section{Recording of physiological signals}

The physiological signals of the both control and experimental groups were recorded before and after a month. The raw ECG signals were recorded using three lead ECG electrodes. The ECG signals were recorded for a period of 15 min using the standard lead II triangle configuration for each participant using Super Spec 32 Machine (RMS, India). The RR signals were also recorded using RMS capacitive probe. The $256 \mathrm{~Hz} /$ channel (RMS, India) was set as the sampling frequency. The power-line interference signals $(50 \mathrm{~Hz})$ were eliminated by selecting notch filter in the machine while recording. Along with these signals, the systolic and diastolic pressures of the participants were measured with the help of a multiparameter monitor. 


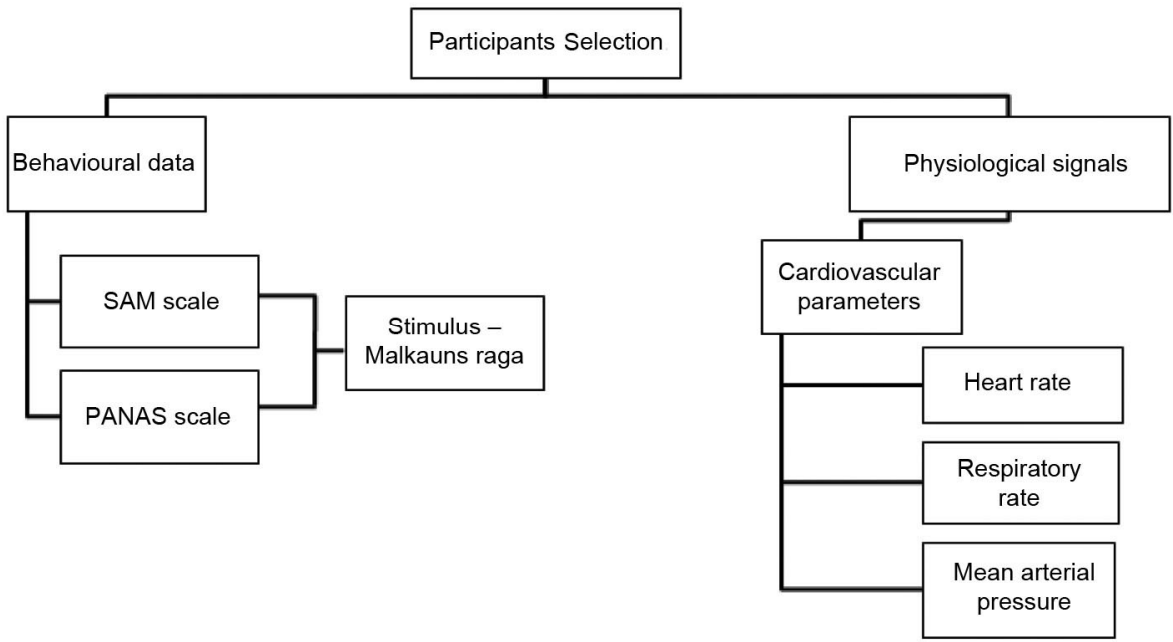

Figure 1. Methodology flow chart.

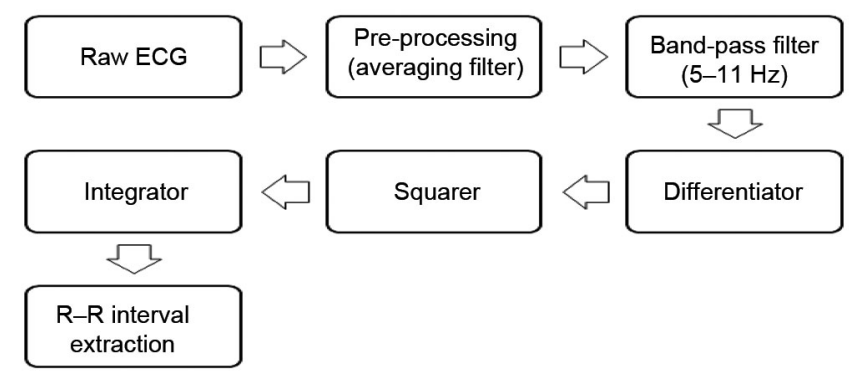

Figure 2. Flow chart for heart rate detection.

The formula used for calculating MAP was

$$
\mathrm{MAP}=\frac{[\mathrm{SBP}+2(\mathrm{DBP})]}{3},
$$

where SBP is the systolic blood pressure and DBP is the diastolic blood pressure.

\section{Data processing}

\section{Detecting heart rate}

For heart rate signal analysis, the acquired ECG signal was pre-processed using moving-average filter of order 5 . The PAN TOMPKINS algorithm was used in the detection of heart rate ${ }^{21,22}$. Figure 2 shows the schematic block diagram of the algorithm. The algorithm includes a bandpass filter, differentiator, squaring and integration to extract the $\mathrm{R}-\mathrm{R}$ interval which gives a measure of the heart rate. The QRS complex from ECG signal is obtained using a band-pass filter of $5-11 \mathrm{~Hz}$ cut-off. The filtered signal is then differentiated to emphasize the QRS complex, as QRS is a high-frequency component in an ECG signal; the high slope components present in the signal connected to the QRS complex are then improved. Within the duration of a single QRS complex, multiple peaks are exhibited by the output of derivative operation. Positive results are obtained by the squaring operation. The algorithm smoothens the output through movingwindow integration filter for the experimental duration (15 min) of the selected epoch. The heart rate is extracted for both experimental and control groups.

The time-frequency of the ECG signal is visualized using short-time Fourier transform (STFT). STFT is used to analyse non-stationary signals like ECG. STFT is a technique which divides the signal into short timeintervals and then finds the Fourier transform of each segment. The time-frequency visualization provides changes in frequency with respect to the selected epoch. In this analysis, selecting an appropriate window becomes important; if the window is too short, the frequency resolution is reduced and if the window is too long, it will reduce the time localization ${ }^{23}$. The significant parameters in STFT are frequency bins, time steps, window type and window length. Here, the frequency bin and time steps were set to 512 and 32 respectively. The window type used was Hanning and window length was fixed at 64 . Figure $3 a-e$ displays $10 \mathrm{sec}$ epoch of $15 \mathrm{~min}$ data.

The methods followed for feature extraction (heart rate) for the experimental group are discussed below.

Figure $3 a$ shows the raw ECG signal for the experimental group after exposure to music for a month. Figure $3 b$ shows the time-frequency representation of the same.

Moving-average filter with rectangular window was selected to pre-process the raw ECG signal. The full width of moving-average window is given by $N=1+2 M$ samples, where $M$ is half-width of moving average and is chosen as 10 .

The calculated heart rate for participants after exposure to music was $78 \mathrm{BPM}$. The same method of processing was used for the control group and heart rate of 99 BPM 


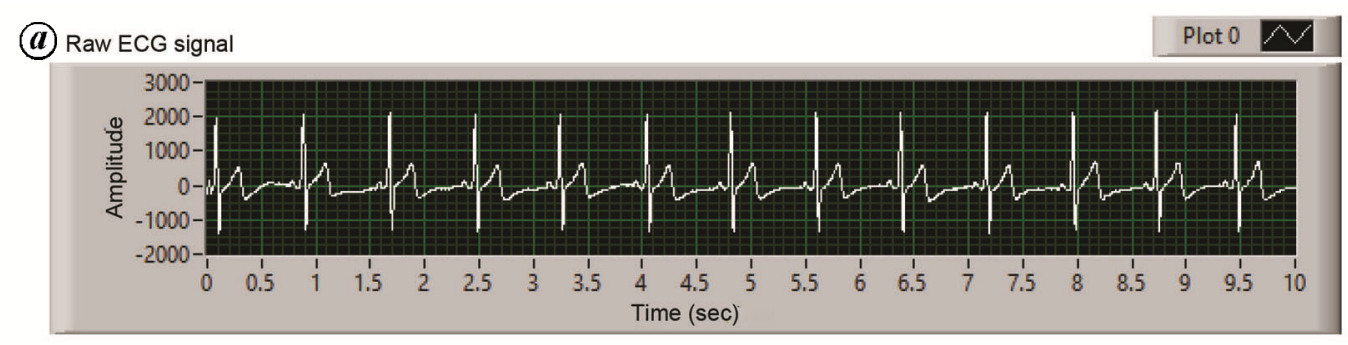

Raw ECG signal
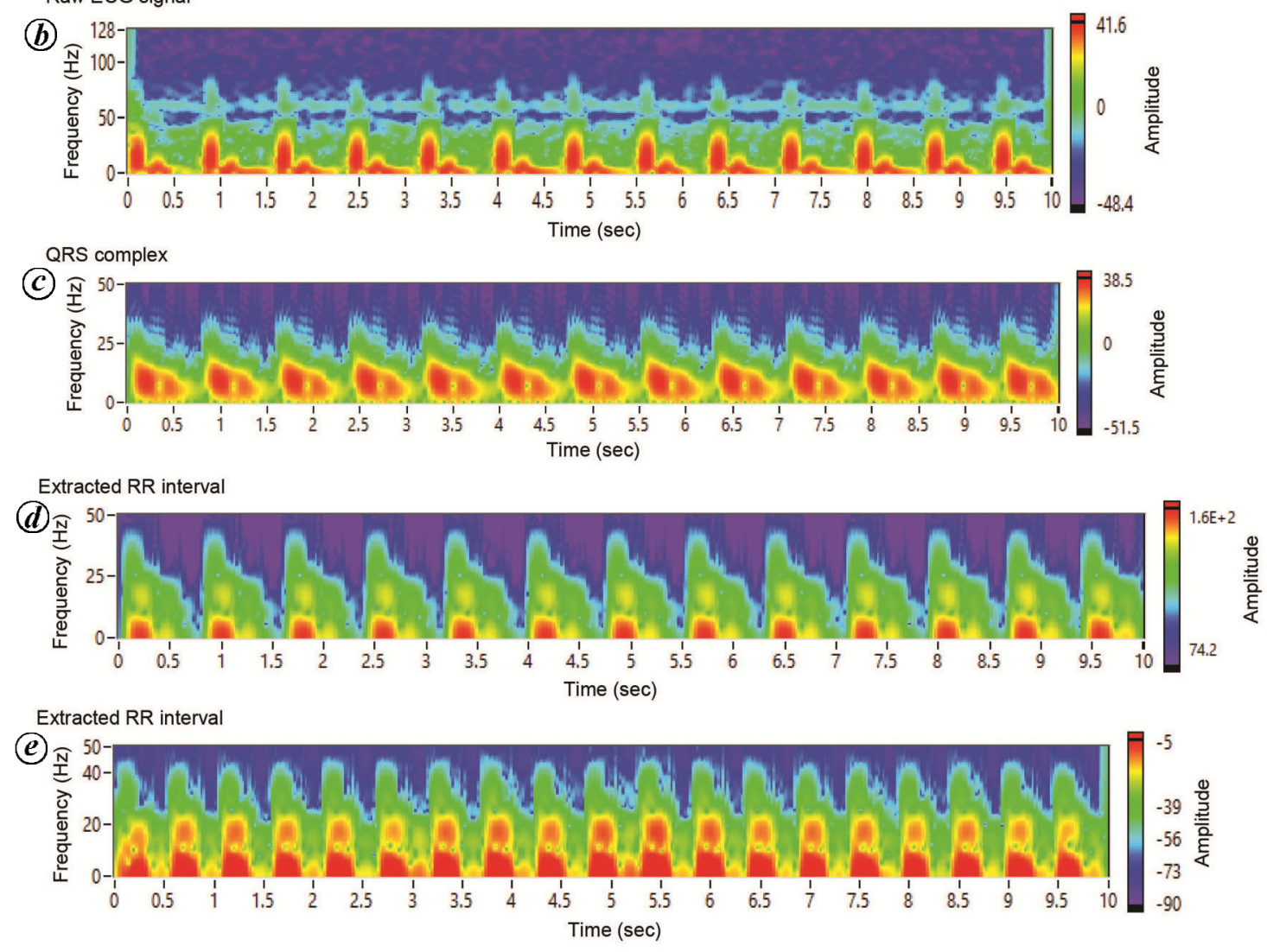

Figure 3. $\boldsymbol{a}$, Raw ECG signal after exposing the experimental group to music for a month (epoch 10 sec). $\boldsymbol{b}$, Time-frequency representation of raw ECG signal after exposing the experimental group to music for a month (epoch 10 sec). $c$, Time-frequency representation of extracted QRS complex, after exposing the experimental group to music for a month (epoch $10 \mathrm{sec}$ ). $\boldsymbol{d}$, Time-frequency distribution using short time Fourier transform (STFT) which eliminates the low-frequency components like $P$ wave and $T$ wave from the ECG wave, and displaying only the QRS wave for experimental group after a month. $\boldsymbol{e}$, Time-frequency distribution using STFT which eliminates the low-frequency components $P$ wave and $T$ wave from the ECG wave, and displaying only the QRS wave for the control group after a month.

was noted. Figure $3 e$ shows the extracted $\mathrm{R}-\mathrm{R}$ interval.

\section{Detecting respiratory rate}

The RR was measured using a capacitive probe using Super Spec 32 machine (RMS, India). The recorded respiratory signals were processed off-line (Figure 4). Figure $5 a$ shows the raw respiratory signal and Figure $5 b$ the time-frequency representation of raw resipratory signal after exposing the experimental group to music for a month. The first step is using moving-average filter of order 25 (Figure $5 c$ ). Figure $5 d$ is the time-frequency representation of the same.
The time-interval between the two peaks gives a measure of the RR.

The processed respiratory signal was fed to the peak detector to extract the peaks of the respiratory waveform. Time-period was calculated to measure the RR and it was found to be $16 / \mathrm{min}$ after exposing the experimental group to music for a month. The same procedure was followed to extract the RR from the control group after a month.

\section{Detecting MAP}

MAP is the average pressure in the arteries of a patient during one cardiac cycle. The DBP and SBP of the patients were measured with the help of a multi-parameter 
monitor. MAP was calculated from eq. (1). The SBP and DBP for each participant in both the groups were recorded for $15 \mathrm{~min}$. Both were measured at every $5 \mathrm{~min}$ interval and so three-pressure values were obtained and mean average was taken for calculating MAP; the selected epoch of measurement was $15 \mathrm{~min}$.

\section{Statistical analysis}

The cardiovascular parameters were measured for both control (pre and post) and experimental group (pre and post). The data measured were normally distributed; so parametric analysis was done to compare the means within (related sample) and between (independent sample) groups. The control and experimental groups of hypertensive participants were the independent variables and their heart rate, RR and MAP were the dependent variables. The scoring for SAM (valence and arousal) and PANAS (positive affect and negative affective scores) were considered as dependant variables, as these scores vary with the rating of the participants. The paired-samples $t$-test was used to compare the means of the same group (pre and post conditions), heart rate, RR and MAP before and after one month. The independent samples $t$-test was used to compare the means of the dependent variables (heart rate, RR and MAP) between control and experimental groups. The alpha value (positive affective scores, twotailed) was less than 0.05 . The analysis was carried out using SPSS 24 for Windows (SPSS Inc, Chicago, USA) for statistical calculations.

\section{Results}

\section{Behavioural data}

As participants listened to music for a short period every day for a month, mood assessment becomes necessary and it was done using the PANAS scale. The positive affective scores of Malkauns raga were significant after listening to music $(P=0.005)$ when compared to before listening, while the negative affective scores were significantly reduced $(P=0.001)$, as in Figure 6 . The results reveal that the Malkauns raga has high positive affect score and low negative affect score, which indicates mood changes among the participants.

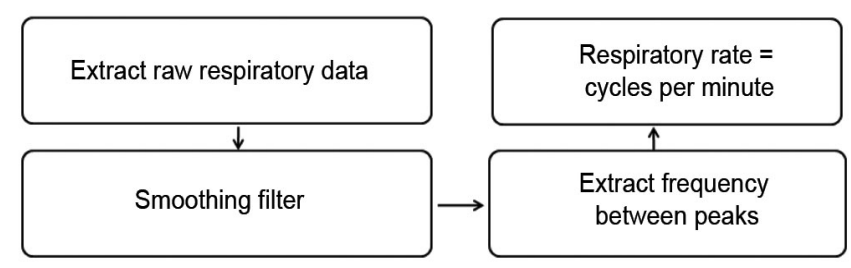

Figure 4. Block diagram for respiratory rate measurement.
The physiological parameters measure bodily health and emotional conditions; the perceived emotions of the participants were measured using SAM scale. The valence and arousal were calculated (Figure 7); the valence for Malkauns raga was $4 \pm 0.1581$ and arousal rating was $2.20 \pm 0.1432$. Based on SAM scores, high valance and low arousal stimulus is shown to enhance the health of the listeners.

\section{Physiological parameters}

The paired-samples $t$-test was used to compare the means of the same group (pre and post conditions), heart rate, RR and MAP before (pre) and after (post) one month. There was no change in heart rate for pre- and postconditions (after one month) of the control group $(t(99)=1.597, P=0.113)$, whereas the heart rate for pre- and post-condition of experimental group was $t(99)=6.669, P<0.0001$ (Figure $8 a)$.

There was significant change in mean RR for pre- and post-conditions of control $(t(99)=2.87, P<0.005)$ as well as experimental $(t(99)=6.359, P<0.0001)$ groups (Figure $8 b$ ).

When MAP was compared, there was no change for pre- and post-conditions of control group $(t(99)=0.911$, $P=0.364)$, whereas for the experimental group $(t(99)=$ $20.250, P<0.0001$ ), MAP was highly significant (Figure $8 c$ ), which indicates that the high valence and low arousal music played has an effect on the heart rate and MAP only.

\section{Comparing control and experimental groups}

The independent-samples $t$-test was used to compare the means of the dependent variables (heart rate, RR and MAP) between control and experimental groups (Figure 9 and Table 1).

\section{Discussion}

Music intervention can improve physiological and psychological health of individuals ${ }^{24,25}$. Music relaxes and enhances the mood of a listener ${ }^{26}$. This study focuses on the influence of music intervention on hypertensive patients using their preferred choice of music, and measures the physiological signals before and after a month of intervention. Music selection becomes an important parameter in studying the effect on physiological signals, as we all react differently to diverse forms of music ${ }^{27}$. In addition, music that can change the mood of a listener along with enhancing the positive affect score will be helpful in alleviating stress. Autonomic response changes with respect to pleasurable experience of musiclistening ${ }^{28,29}$ and is reflected in heart rate parameter ${ }^{30}$. In 

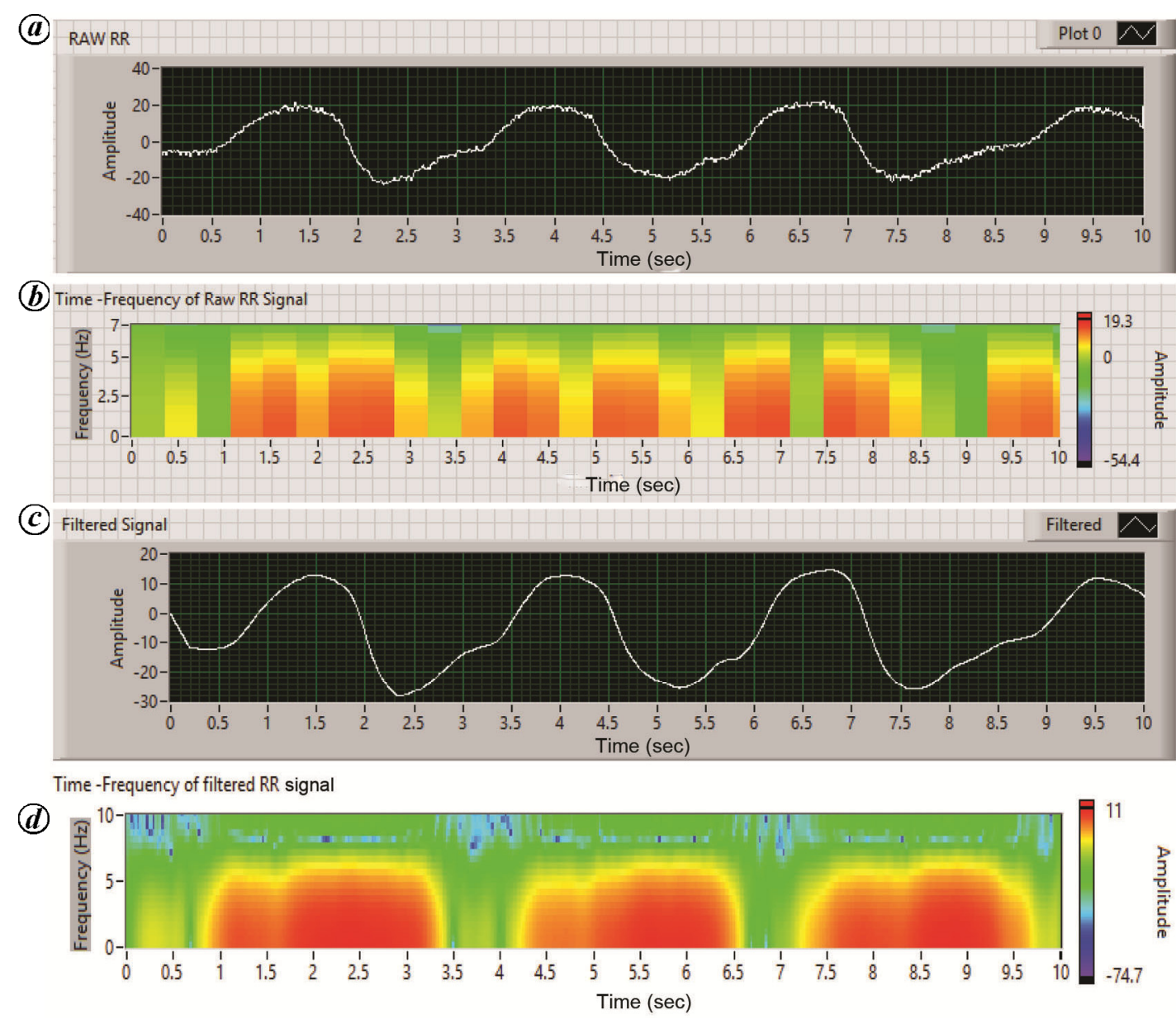

Figure 5. $\boldsymbol{a}$, The raw respiratory waveform of participants after exposure of the experimental group to music for a month. $\boldsymbol{b}$, Time-frequency distribution using STFT technique of the raw respiratory waveform of participants after exposure of experimental group to music for a month. $c$, The processed filtered output using a moving average filter of order 25. $\boldsymbol{d}$, Time-frequency distributor of filtered respiratory signal of the experimental group after exposure to music for a month.
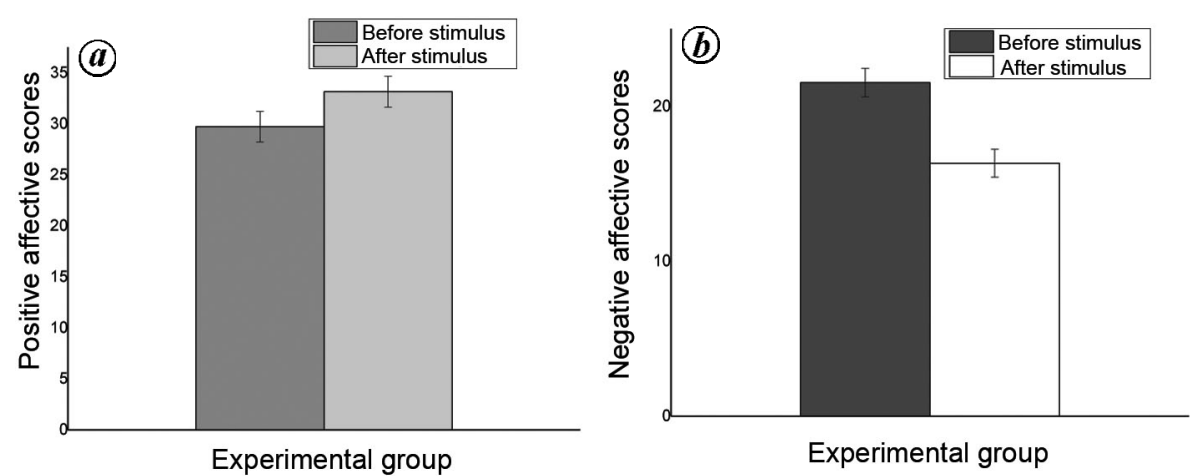

Figure 6. Positive affect and negative affective scores (PANAS) results after listening to Raga Malkauns (stimulus): (a) positive affect score and $(\boldsymbol{b})$ negative affective score.

the present study, the experimental group was provided with musical stimulus (Malkauns raga) and asked to rate their mood using PANAS scale ${ }^{31}$. Along with this, perceived emotion valence (pleasant/unpleasant) and arousal (calm/activation) was measured using SAM scale. Raga
Malkauns was found to have high positive affect score after listening, along with increased pleasant and low arousal scores. This clearly indicates the mood change and low arousal for the participants, and thereby reflects the changes in acquired physiological signals. 


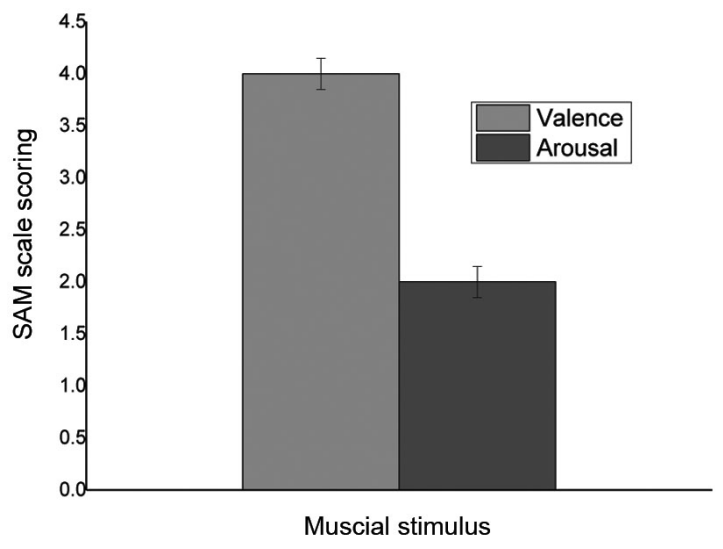

Figure 7. SAM scale scoring after listening to Raga Malkauns stimulus; measured valence and arousal.
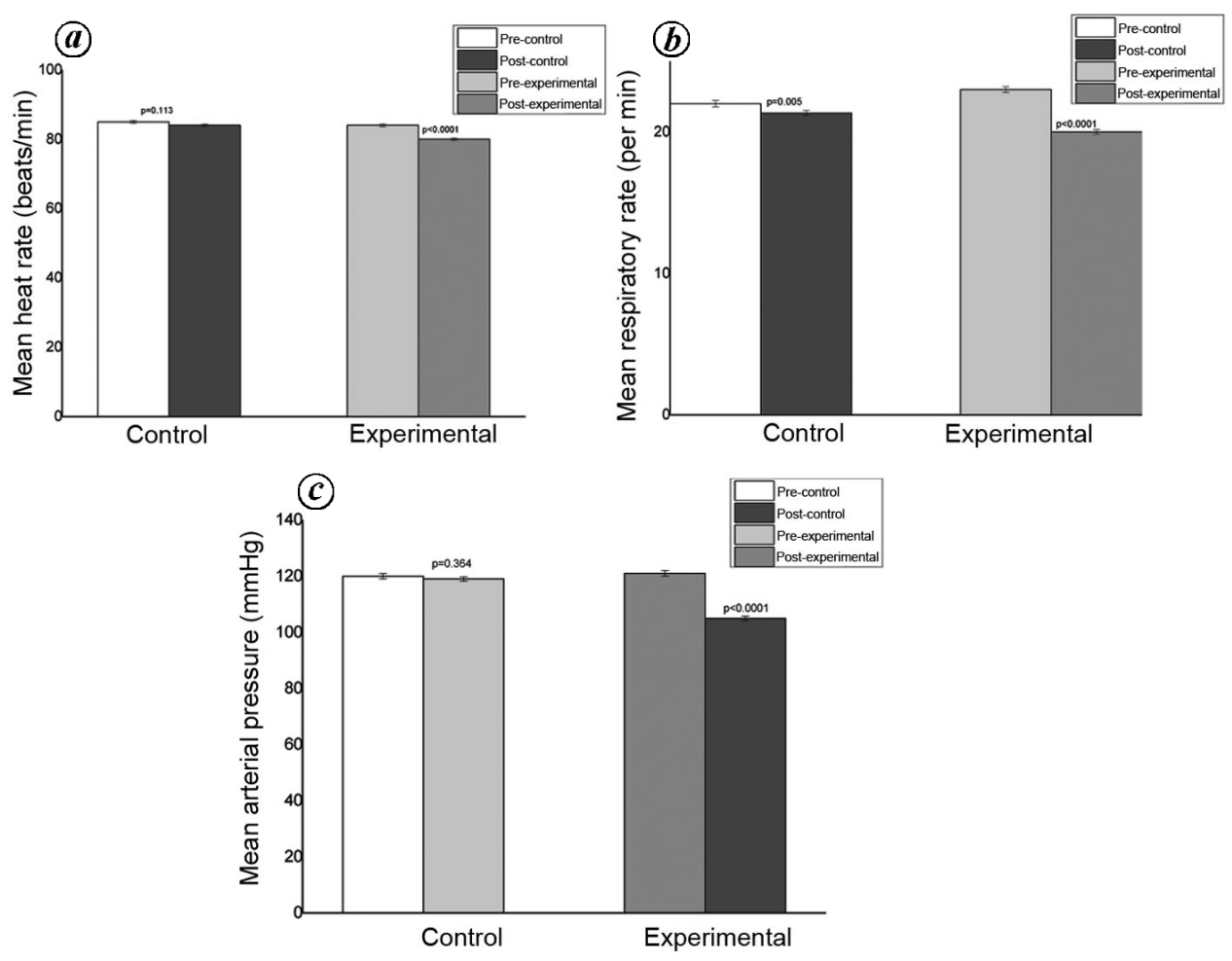

Figure 8. Variations in $(\boldsymbol{a})$ mean heart rate, $(\boldsymbol{b})$ mean respiratory rate and $(\boldsymbol{c})$ mean arterial pressure for control and experimental groups.

Hilz et al..$^{32}$ reported that arousal response influences the cardiovascular response than valence of the played music. Etzel et al. ${ }^{33}$ found that heart rate is elevated by high arousal music, whereas low arousal music had a reverse effect and our results support their findings that heart rate and MAP significantly lower for the experimental group. This was not noted for the control group, even though no significant changes were noted for RR. There was no change in heart rate for pre- and postconditions (after one month) of the control group $(P=0.113)$, whereas for the experimental group it was significantly lower $(P<0.0001)$. There was significant reduction in mean RR for both control $(P<0.005)$ and for experimental $(P<0.0001)$ groups for pre- and postconditions. There was no change in MAP for pre- and post-conditions of control group $(P=0.364)$, whereas for the experimental group $(P<0.0001)$, MAP was significantly lower, which indicates that the high valence and low arousal music played has an effect on heart rate and MAP only. This clearly shows that the selected music intervention activates the parasympathetic nervous system.

Bernardi ${ }^{34}$ used fast music rhythms to increase heart rate, $\mathrm{BP}$ and ventilation which elucidate the high arousal responses. On the other hand, slower music rhythms lower the heart rate, BP and ventilation. Siritunga et al..$^{35}$ 

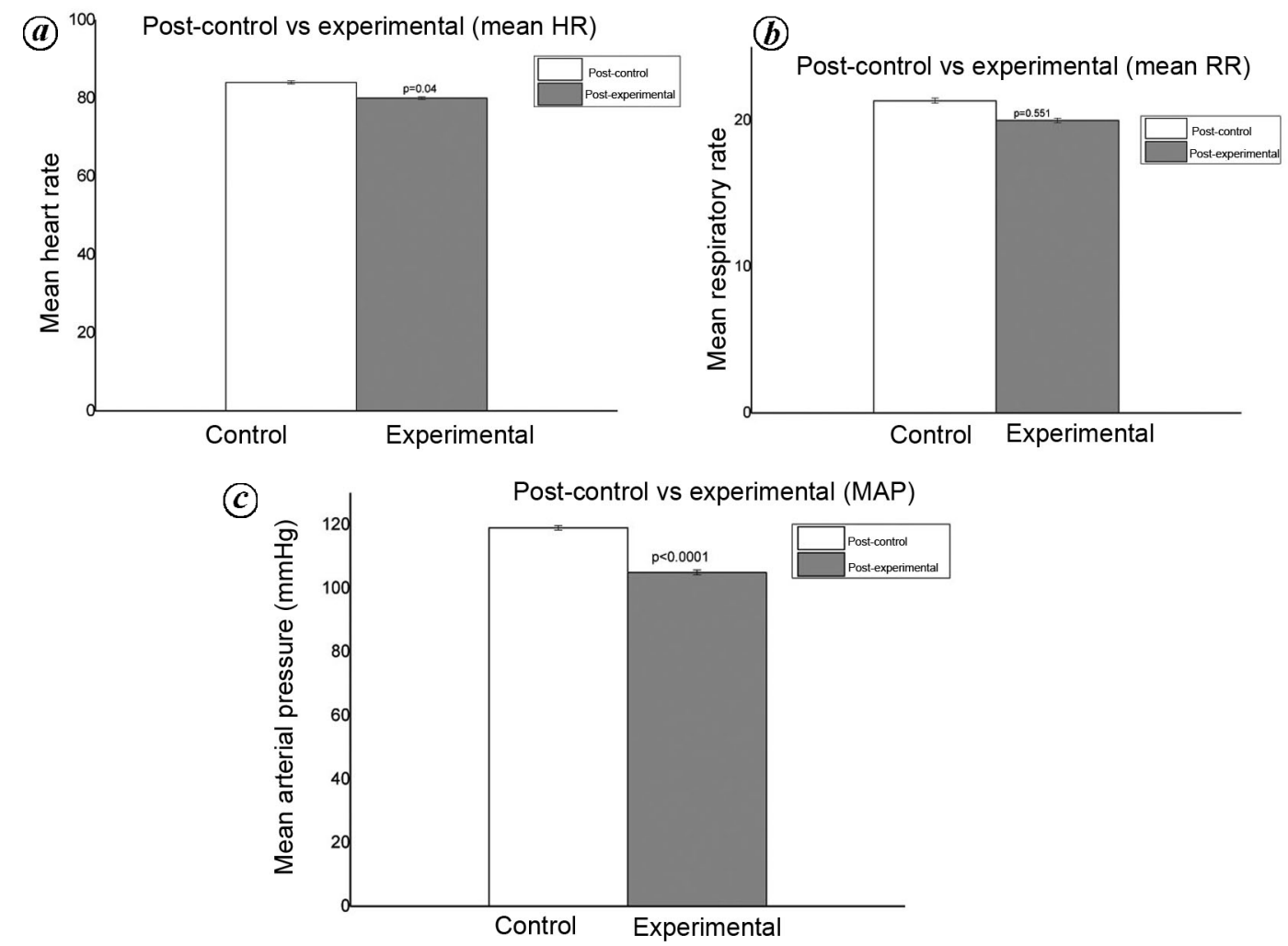

Figure 9. Variations in $(\boldsymbol{a})$ mean heart rate, $(\boldsymbol{b})$ mean respiratory rate and $(\boldsymbol{c})$ mean arterial pressure for control and experimental groups.

Table 1. Comparison of heart rate, mean arterial pressure (MAP) and respiratory rate between control and experimental groups

\begin{tabular}{lccccc}
\hline Pre-heart rate groups & Pre-respiratory rate & Pre-MAP & Post-heart rate & Post-respiratory rate & Post-MAP \\
\hline$t(198)=1.478$, & $t(198)=0.710$, & $t(198)=-1.258$, & $t(198)=2.360$, & $t(198)=0.710$, & $t(198)=12.427$, \\
$P=0.141$ & $P=0.479$ & $P=0.210$ & $P=0.019$ & $P=0.479$ & $P<0.0001$ \\
\hline
\end{tabular}

used Indian classical music to study asymptomatic individuals and concluded that DBP, SBP, pulse rate and RR were reduced. Music intervention group (experimental) showed significant decrease in MAP and heart rate after listening to music; this was not noted for the control group before and after a month. These results are concurrent with the findings of Bernardi and Siritunga et al. The music intervention was given for a period of one month; as this had reduced the heart rate and MAP. If this intervention is extended to another six months, the results would be further improved. The changes were noted only for heart rate and MAP and not for RR. Perhaps using different music stimulus would yield reduction in RR.

\section{Conclusion}

In the present study we evaluate the response of music interventions on heart rate, MAP and RR. The heart rate and MAP of the control group did not vary greatly under the influence of the drugs prescribed. On the other hand, the difference in heart rate and MAP was significantly lower after listening to music for a period of one month. Thus the study concludes that the participants preferred high valence and low arousal music, which yielded better results in activating the parasympathetic nervous response, thereby lowering the heart rate and MAP. Music intervention along with drugs will pave way for the reduction in sympathetic activation rather than the drug treatment alone. To support this further, the cardiovascular parameters while listening to music will be studied to know the immediate effect of music.

Ethical statement: Ethical clearance was obtained from the Institutional Human Ethics Committee, Chettinad Academy of Research and Education, Chennai. The registration number is 9908030 .

Conflict of interest: The authors declare no conflict of interest. 
1. Gupta, M., Brister, S. and Verma, S., Is South Asian ethnicity an independent cardiovascular risk factor? Can. J. Cardiol., 2006, 22(3), 193-197.

2. Mansia, G. et al., ESH-ESC Guidelines for the management of arterial hypertension: the task force for the management of arterial hypertension of the European Society of Hypertension (ESH) and of the European Society of Cardiology (ESC). Blood Pressure, 2007, 16(3), 135-232.

3. Angioli, R. et al., Use of music to reduce anxiety during office hysteroscopy: prospective randomized trial. J. Minim. Invas. Gynaecol., 2014, 21(3), 454-459.

4. Kamioka, H. et al., Effectiveness of music therapy: a summary of systematic reviews based on randomized controlled trials of music interventions. Patient Prefer. Adher., 2014, 8, 727-754.

5. Colley, A., Young people's musical taste: relationship with gender and gender-related traits 1. J. Appl. Soc. Psychol., 2008, 38(8), 2039-2055.

6. North, A. C., Hargreaves, D. J. and Hargreaves, J. J., The uses of music in everyday life. Music Percept.: Interdisci. J., 2004, 22(1), 41-77.

7. Wells, A. and Hakanen, E., The emotional use of popular music by adolescents. Journalism Q., 1991, 68(3), 445-454.

8. Geethanjali, B., Adalarasu, K., Mohan, J. and Seshadri, N. P. G., Music induced brain functional connectivity using EEG sensors: a study on Indian music. IEEE Sensors J., 2018, 19(4), 14991507.

9. Geethanjali, B., Adalarasu, K., Jagannath, M. and Rajasekaran, R., Influence of pleasant and unpleasant music on cardiovascular measures and task performance. Int. J. Biomed. Eng. Technol., 2016, 21(2), 128.

10. Sarkamo, T. et al., Music listening enhances cognitive recovery and mood after middle cerebral artery stroke. Brain, 2008, 131(3), 866-876.

11. Sacks, O., Musicophilia: Tales of Music and Brain, Knopf, New York, USA, 2007, pp. 56-57.

12. Bradt, J., Dileo, C. and Potvin, N., Music for stress and anxiety reduction in coronary heart disease patients. Cochrane Database Syst. Rev., 2013, 12, 5-6.

13. Bonde, L. O., Health musicing-music therapy or music and health? A model, empirical examples and personal reflections. Music Arts Action, 2011, 3(2), 120-140.

14. Bradt, J., Dileo, C. and Potivin, N., Music for stress and anxiety reduction in coronary heart disease patients. Cochrane Database Syst. Rev., 2013, 12, 44-47.

15. AMTA, Definition and quotes about music therapy, American Music Therapy Association (on-line), 2018; http://www. musictherapy.org/about/quotes/ (accessed on 5 December 2018).

16. Swaminathan, S. and Schellenberg, E., Current emotion research in music psychology. Emot. Rev., 2015, 7(2), 189-197.

17. Deshmukh, A., Sarvaiya, A., Seethalakshmi, R. and Nayak, A., Effect of Indian classical music on quality of sleep in depressed patients: a randomized controlled trial. Nordic J. Music Ther., 2009, 18(1), 70-78.

18. Sarkar, J. and Biswas, U., Indian classical ragas to cure diseases. Int. J. Adv. Sci. Res., 2015, 1(1), 9-13.
19. Bradley, M. and Lang, P., Measuring emotion: the self-assessment manikin and the semantic differential. J. Behav. Ther. Exp. Psychiatry, 1994, 25(1), 49-59.

20. Watson, D., Clark, L. and Tellegen, A., Development and validation of brief measures of positive and negative affect: The PANAS scales. J. Pers. Soc. Psychol., 1988, 54(6), 1063-1070.

21. Pan, J. and Tompkins, W., A real-time QRS detection algorithm. IEEE Trans. Biomed. Eng., 1985, BME-32(3), 230-236.

22. Menon, S., Geethanjali, B., Guhan Seshadri, N., Muthumeenakshi, S. and Nair, S., Evaluating the induced emotions on physiological response. Lect. Notes Electr. Eng., 2018, 490, 211-220.

23. Balasubramanian, G., Kanagasabai, A., Mohan, J. and Seshadri, $\mathrm{N}$., Music induced emotion using wavelet packet decompositionan EEG study. Biomed. Signal Proc. Control, 2018, 42, 115-128.

24. Koelsch, S., Towards a neural basis of music-evoked emotions. Trends Cogn. Sci., 2010, 14(3), 131-137.

25. Cooke, M., Irby, D. M. and O'Brien, B. C., Educating Physicians: A Call for Reform of Medical School and Residency (Vol. 16), John Wiley, 2010.

26. Lonsdale, A. and North, A., Why do we listen to music? a uses and gratifications analysis. Br. J. Psychol., 2011, 102(1), 108-134.

27. Geethanjali, B., Adalarasu, K., Jagannath, M. and Rajasekaran, R., Enhancement of task performance aided by music. Curr. Sci., 2016, 111(11), 1794-1801.

28. Blood, A., Zatorre, R., Bermudez, P. and Evans, A., Emotional responses to pleasant and unpleasant music correlate with activity in paralimbic brain regions. Nature Neurosci., 1999, 2(4), 382387.

29. Blood, A. and Zatorre, R., Intensely pleasurable responses to music correlate with activity in brain regions implicated in reward and emotion. Proc. Natl. Acad. Sci. USA, 2001, 98(20), 11818 11823.

30. Panksepp, J. and Bernatzky, G., Emotional sounds and the brain: the neuro-affective foundations of musical appreciation. Behav. Proc., 2002, 60(2), 133-155.

31. Miller, M. and Fry, W., The effect of mirthful laughter on the human cardiovascular system. Med. Hypotheses, 2009, 73(5), 636-639.

32. Hilz, M. et al., Music induces different cardiac autonomic arousal effects in young and older persons. Auton. Neurosci., 2014, 183, 83-93.

33. Etzel, J., Johnsen, E., Dickerson, J., Tranel, D. and Adolphs, R., Cardiovascular and respiratory responses during musical mood induction. Int. J. Psychophysiol., 2006, 61(1), 57-69.

34. Bernardi, L., Cardiovascular, cerebrovascular, and respiratory changes induced by different types of music in musicians and nonmusicians: the importance of silence. Heart, 2005, 92(4), 445452.

35. Siritunga, S., Wijewardena, K., Ekanayaka, R. and Mudunkotuwa, P., Effect of music on blood pressure, pulse rate and respiratory rate of asymptomatic individuals: a randomized controlled trial. Health, 2013, 5(4), 59-64.

Received 30 March 2019; accepted 30 October 2019

doi: $10.18520 / \mathrm{cs} / \mathrm{v} 118 / \mathrm{i} 4 / 612-620$ 